\title{
Vicarious trauma and secondary traumatic stress in health care professionals
}

\author{
Nicole A Guitar, Monica L Molinaro \\ Faculty Reviewer: Paul Frewen, PhD (Department of Psychology)
}

\section{ABSTRACT}

Three-quarters of Canadians are exposed to a traumatic event sufficient to cause psychological trauma in their lifetime. In fact, post-traumatic stress disorder is a global health issue with a prevalence as high as $37 \%$. Health care professionals trained to provide mental health treatment for these individuals are at risk of developing vicarious trauma and secondary traumatic stress, both of which result in adverse symptoms for the health care provider that often mimic post-traumatic stress disorder (PTSD). Vicarious trauma develops over time as the clinician is continually exposed to their clients' traumatic experiences, while clinicians experiencing secondary traumatic stress begin to experience the symptoms of PTSD due to secondary exposure of the traumatic event. Both vicarious trauma and secondary traumatic stress cause mental, physical, and emotional issues for health care professionals that include burnout and decreased self-worth. Health care systems and administration should aim to develop training and professional education for health care providers. This review will emphasize what factors lead to the development of vicarious trauma and secondary traumatic stress, and what aids or supports can be implemented to treat the symptoms. The implications for policy development and training will be discussed.

Based on current statistics, 1 in 5 Canadians will experience a mental health or addiction problem in their lifetime, and $50 \%$ of individuals over 40 years of age will experience some form of mental illness. ${ }^{1}$ Mental illness is one of the leading causes of disability in Canada. ${ }^{2-4}$ There are a variety of health care professionals trained to provide mental health treatment to patients who seek it; however, these health care professionals may be vicariously affected by their clients while providing care. This paper will discuss the concepts of vicarious trauma (VT) and secondary traumatic stress (STS) in health care providers, what factors lead to the development of VT and STS, and what aids or supports can be implemented to assist individuals affected by VT and STS.

Vicarious trauma refers to negative changes that can occur in a health care provider that specifically alter their beliefs regarding themselves, others, and their worldview. ${ }^{5,6}$ VT results from exposure to individuals who have undergone traumatic experiences. ${ }^{5,6}$ The concept of VT was developed using constructivist theories which posit that learning is a constructive process where people actively construct their own subjective representations of subjective reality. ${ }^{7}$ Clinicians can experience VT when exposed to their patients' traumatic experiences which triggers negative beliefs about safety, power, independence, esteem, and intimacy. ${ }^{6}$ VT can also lead to "decreased motivation, efficacy and empathy". ${ }^{7}$ Typically, VT develops over time as an individual is continually exposed to their clients' experiences, and often manifests mentally while presenting as symptoms that align with post-traumatic stress disorder (PTSD). ${ }^{6}$

Secondary traumatic stress, often referred to incorrectly as "compassion fatigue", ${ }^{8}$ describes a set of symptoms similar to those of PTSD, $, 5,89$ such as "exhaustion, hypervigilance, avoidance, and numbering". ${ }^{8}$ STS can occur in health care professionals, family members, friends, and caregivers of individuals who have experienced traumatic events, and who often have post-traumatic stress themselves. ${ }^{5,8-10}$ Individuals experiencing STS do not experience the traumatic events first-hand; instead, due to secondary exposure of the traumatic event, they begin to experience the symptoms of PTSD. . $^{5-10}$

Vicarious trauma and secondary traumatic stress have many similarities and while the two terms are meant to describe different experiences, they are often used interchangeably to represent the same phenomenon. ${ }^{5}$ However, VT and STS represent two distinct experiences and they apply to different populations. ${ }^{10}$ STS can be experienced by multiple sets of individuals, while vicarious trauma applies only to those individuals in direct care positions, such as first responders, health care providers, and social workers. ${ }^{10} \mathrm{STS}$ and VT can be clearly differentiated by examining the length of manifestation of these two disorders. STS typically manifests for a shorter period of time compared to VT. ${ }^{5}$ For example, STS can occur in an emergency response worker or response team, who has a limited exposure time to the individual experiencing trauma, which differs from the development of VT and "the experience of a psychotherapist bearing witness to years of sexual abuse". 5

Individuals who provide care or treatment for trauma-related incidents are at a significantly higher risk for the development of STS and VT, since the symptoms of these disorders present themselves after "exposure to traumatic experiences described by their clients". ${ }^{10}$ There are risk factors specific to the development of VT and STS. Previous studies have reported that an individual's personal history of trauma is directly linked to the development of VT. ${ }^{5,1-14}$ Additionally, the amount of time spent with patients or clients who have experienced trauma, and the proportion of trauma cases that a health care provider treats, are predictors for the development of VT. ${ }^{15}$ Personal trauma history and the quantity of exposures to different patients and their traumatic experiences are significantly linked to the development of STS..$^{5,1-15}$ Health care providers treat- 
ing PTSD with cognitive therapies are at greater risk for VT and STS because many psychological treatment methods require patients to provide a detailed account of their traumatic experience. ${ }^{8}$ By providing therapy for individuals that can manage symptoms of their PTSD, the health care provider is at a greater risk of developing STS themselves. ${ }^{8}$

Both secondary traumatic stress and vicarious trauma manifest in particular ways. In a study conducted by Baird \& Kracen (2006), it was found that VT "is associated with disruptions to schemas" in five areas: (1) safety, (2) trust, (3) esteem, (4) intimacy, and (5) control, each of which represent a crucial "psychological need". ${ }^{5}$ Schemas represent patterns of thought that organize categories of information. ${ }^{5}$ VT negatively affects these five important schemas, and can create a health care provider's perception that there is a lack of safety in their own world. ${ }^{5,7}$ In contrast, STS often manifests physically as "exhaustion, hypervigilance, avoidance, and numbing"5 and is specifically associated with PTSD.

VT and STS can be mediated or prevented in multiple ways. Health care systems and administration can aim to develop training and professional education for VT and STS in health care providers, and seek to evaluate existing programs for efficacy and areas of improvement. ${ }^{5}$ Specifically, health care professionals need resources and improved clinical training, with additional resources and treatment availability for individuals who have been affected by VT or STS. ${ }^{10}$ Health administrators should take actions to decrease health care provider's caseloads, increase leave time for health care professionals, increase supervision and staff support, and increase the development and provision of additional mental health resources. ${ }^{10,16}$ Health care providers should ensure that they are cognizant of the symptoms of VT and STS and that they are participating in their own self-care, maintaining their personal and professional obligations and activities, and reflecting on any cognitive or physical changes they may experience..$^{10}$

VT and STS pose many mental, physical, and emotional problems for health care professionals, including burnout, decreased self-worth and low morale. ${ }^{1718}$ This can lead to higher staff turnover, as well as decreased productivity amongst health care professionals. ${ }^{17}$ Nevertheless, recent research suggests that in a small number of cases, health care providers affected by VT and STS may develop vicarious resilience in the form of strength, growth, and empowerment arising from an optimism for hope and change. ${ }^{16}$ This idea has implications for policy development and health care practices that encourage health care providers to share both positive and negative work experiences as part of the prevention and treatment of VT and STS.

\section{REFERENCES}

1. Smetanin P, Stiff D, Briante C, et al. The life and economic impact of major mental illnesses in Canada: 2011-2041. Prepared for the Mental Health Commission of Canada. 2011. Toronto: RiskAnalytica.

2. Lim K, Jacobs P, Ohinmaa A, et al. A new population-based measure of the burden of mental illness in Canada. Chronic Disease Can, 2008; 28: 92-8.

3. Mental Health Commission of Canada [Internet]. Ottawa (ON): Mental
Health Commission of Canada; c2014. Why investing in mental health will contribute to Canada's economic prosperity and to the sustainability of our health care system; c2014 [cited 2017 March 20]. Available from: http://www.mentalhealthcommission.ca/English/media/3104

4. Institute for Health Metrics and Evaluation [Internet] Seattle (WA): Institute for Health Metrics and Evaluation; c2015. Global Burden of Diseases, Injuries, and Risk Factors Study, 2013; c2015. [cited 2017 March 20]. Available from: http://www.healthdata.org/data-visualization/gbd-compare

5. Baird K, Kracen AC. Vicarious traumatization and secondary traumatic stress: A research synthesis. Counsl Psych Quart 2006; 19(2):181-188.

6. Pearlman LA, Saakvitne KW. Trauma and the therapist: Countertransferance and vicarious traumatization in psychology with incest survivors. New York: W. W. Norton; 1995.. 451 p.

7. McCann IL, Pearlman MM. Vicarious traumatization: A framework for understanding the psychological effects of working with victims. $J$ Traumatic Stress 1990; 3:131-149.

8. Figley C. Compassion Fatigue. New York: Bruner/Mazel; 1995. 292 p.

9. Stamm B (Eds). Secondary traumatic stress: Self-care issues for clinicians, researchers, and educators. Lutherville MD: Sidran Press; 1999. $332 \mathrm{p}$.

10. Elwood LS, Mott J, Lohr JM, et al. Secondary trauma symptoms in clinicians: A critical review of the construct, specificity, and implications for trauma-focused treatment. Clinical Psych Rev. 2011; 31:25-36.

11. Camerlengo $\mathrm{H}$. The role of coping style, job-related stress, and personal victimization history in vicarious traumation of professionals who work with abused youth. Doctoral dissertation, Rutgers University, New Jersey.

12. Dickes SJ. Treating sexually abused children versus adults: An exploration of secondary traumatic stress and vicarious traumatization among therapists. Doctoral dissertation, California School of Professional Psychology, Fresno.

13. Pearlman LA, MacIan PS. Vicarious traumatization: An empirical study of the effects of trauma work on trauma therapists. Prof Psych Resea Pract 1995; 26:558-565.

14. Trippany RL. Predictors of vicarious traumatization: Female therapists for adult survivors versus female therapists for child survivors of sexual victimization. 2000. Doctoral dissertation: University of Alabama.

15. Brady, JL, Guy JD, Poelstra PL, et al. Vicarious traumatization, spirituality, and the treatment of sexual abuse survivors: A national survey of women psychotherapists. Professional Psychology, Research and Practice. 1999; 30(4):386-393.

16. Puvimanasinghe T, Denson LA, Augoustinos M, et al. Vicarious resilience and vicarious traumatization: Experiences of working with refugees and asylum seekers in South Austrailia. Transcultu Psych. 2015; 52(6):743-765.

17. Showalter SE. Compassion fatigue: What is it? Why does it matter? Recognizing the symptoms, acknowledging the impact, developing the tools to prevent compassion fatigue and strengthen the professional already suffering from the effects. Am J Hospice Palla Med. 2011; 27(4):239-242.

18. Simon CE, Pryce JG, Roff LL, et al. Secondary traumatic stress and oncology social work: Protecting compassion from fatigue and comprising the worker's worldview. J of Psychosocial Oncology. 2005; 23(4):1-15. 